Burchard, CP, Soares, RG, Vargas, VC, Ilha, PV \& Ruppenthal, R (2020). Analysis of the health theme in the base nacional comum curricular. Research, Society and Development, 9(7): 1-14, e509974457.

\title{
Análise da temática saúde na base nacional comum curricular
} Analysis of the health theme in the base nacional comum curricular Análisis del tema de la salud en la base nacional comum curricular

Recebido: 12/05/2020 | Revisado: 13/05/2020 | Aceito: 14/05/2020 | Publicado: 24/05/2020

\section{Camila Pereira Burchard}

ORCID: http://orcid.org/0000-0001-8567-7130

Universidade Federal do Pampa, Brasil

E-mail: camila.burchard@gmail.com

Renata Godinho Soares

ORCID: http://orcid.org/0000-0002-2386-2020

Universidade Federal do Pampa, Brasil

E-mail: renatasoares1807@gmail.com

Verônica de Carvalho Vargas

ORCID: http://orcid.org/0000-0002-0269-4021

Universidade Federal do Pampa, Brasil

E-mail: veronicadecarvalhovargas@gmail.com

Phillip Vilanova Ilha

ORCID: http://orcid.org/0000-0002-4433-0349

Universidade Federal do Pampa, Brasil

E-mail: phillipilha@unipampa.edu.br

Raquel Ruppenthal

ORCID: http://orcid.org/0000-0003-1301-4260

Universidade Federal do Pampa, Brasil

E-mail: raquelruppenthal@unipampa.edu.br

\section{Resumo}

A presente pesquisa teve como principal objetivo verificar a presença da temática saúde na BNCC. O estudo é uma análise documental de fragmentos do texto relativos ao tema saúde e palavras-chave referentes a mesma. A análise ocorreu em duas etapas: primeiro verificou-se a palavra "saúde", posteriormente verificou-se às palavras saudável, saudáveis, bem-estar, 
qualidade de vida, prevenção e tratamento médico. Como resultados, de uma visão geral da BNCC, a temática foi encontrada com maior concentração nos textos relacionados ao Ensino Fundamental, principalmente nas componentes curriculares de Ciências e Educação Física. Percebe-se que a perspectiva de saúde abordada na componente de ciências é extremamente biológica e com enfoque na diminuição dos riscos à doença. Com relação a Educação Física a perspectiva de saúde adotada faz referência a estética e cuidado do corpo, não levando em consideração a preocupação com questões ambientais e sociais que podem interferir na saúde da população. Considera-se a necessidade de que as escolas discutam e incrementem seus currículos com esse tópico, como também faz-se importante que as universidades e mantenedoras promovam ações que dêem atenção especial na produção de material e/ou sequências didáticas que possam ser implementadas na escola básica, bem como sirvam de apoio aos professores.

Palavras-chave: Saúde; Currículo; Ensino fundamental.

\begin{abstract}
The present research had as main objective to verify the presence of the health theme in BNCC. The study is a documentary analysis of fragments of the text related to the health theme and keywords related to it. The analysis took place in two stages: first, the word "health" was verified, then the words healthy, healthy, well-being, quality of life, prevention and medical treatment were verified. As a result, from an overview of BNCC, the theme was found with greater concentration in texts related to Elementary Education, mainly in the curricular components of Science and Physical Education. It is noticed that the health perspective addressed in the science component is extremely biological and focused on reducing the risks to the disease. Regarding Physical Education, the adopted health perspective refers to aesthetics and body care, without taking into account the concern with environmental and social issues that may interfere with the population's health. It is considered the need for schools to discuss and increase their curricula on this topic, as well as it is important that universities and sponsors promote actions that give special attention to the production of material and / or didactic sequences that can be implemented in the school. basic education, as well as supporting teachers.
\end{abstract}

Keywords: Health; Curriculum; Elementary school.

\title{
Resumen
}

La presente investigación tuvo como objetivo principal verificar la presencia del tema de 
salud en BNCC. El estudio es un análisis documental de fragmentos del texto relacionados con el tema de la salud y palabras clave relacionadas. El análisis se realizó en dos etapas: primero se verificó la palabra "salud", luego se verificaron las palabras saludable, saludable, bienestar, calidad de vida, prevención y tratamiento médico. Como resultado, a partir de una visión general de BNCC, el tema se encontró con mayor concentración en textos relacionados con la educación primaria, principalmente en los componentes curriculares de la ciencia y la educación física. Se observa que la perspectiva de salud abordada en el componente científico es extremadamente biológica y se centra en reducir los riesgos para la enfermedad. Con respecto a la educación física, la perspectiva de salud adoptada se refiere a la estética y al cuidado del cuerpo, sin tener en cuenta la preocupación por los problemas ambientales y sociales que pueden interferir con la salud de la población. Se considera la necesidad de que las escuelas discutan y aumenten sus planes de estudio sobre este tema, así como también es importante que las universidades y los patrocinadores promuevan acciones que presten especial atención a la producción de material y / o secuencias didácticas que se pueden implementar en la escuela. educación básica, además de apoyar a los docentes.

Palabras clave: Salud; Currículum; Enseñanza fundamental.

\section{Introdução}

A saúde é parte fundamental na vida do ser humano. Nosso corpo se mantém em atividades diárias de acordo com as condições de saúde de nossas células. Para a Organização Mundial de Saúde (OMS, 1946) "A saúde é um estado de completo bem-estar físico, mental e social, e não consiste apenas na ausência de doença ou de enfermidade". No entanto, o conceito de saúde vai além dessa definição, contemplando também às nossas relações interpessoais com e no ambiente que ocupamos envolvendo muitas vezes a mídia e o nosso processo de escolarização.

Devido à complexidade e importância do tema saúde, entendeu-se a necessidade de verificar como o tema é abordado no documento norteador do currículo - a Base Nacional Comum Curricular (BNCC). Visto que, a saúde, enquanto prática escolar está presente na Educação Básica desde o século XIX, sempre ligada ao fato de hábitos de higiene, e na não disseminação de doenças (Barbi \& Neto, 2017).

O marco inicial relacionado a saúde na escola é a promulgação da lei 5.692/71 (Brasil, 1971), conhecida como a Lei de Diretrizes e Bases da Educação de 1971 (LDB), que instituiu que temas da saúde deveriam ser desenvolvidos de maneira compulsória nos 
currículos escolares de todos os estabelecimentos de ensino do Brasil, por meio dos "programas de saúde" (Monteiro \& Bizzo, 2015). Dessa forma, o tema saúde passa a ser obrigatório no currículo escolar. O Art. $7^{\circ}$ daquela LDB define que "será obrigatória a inclusão de Educação Moral e Cívica, Educação Física, Educação Artística e Programas de Saúde nos currículos plenos dos estabelecimentos de $1^{\circ}$ e $2^{\circ}$ graus” (Brasil, 1971). Percebe-se claramente um viés higienista para a abordagem da temática saúde na escola nesse período.

Em 1997, os Parâmetros Curriculares Nacionais (PCN), um documento orientador para as escolas, propõe uma nova abordagem: a promoção da saúde ocorre, numa perspectiva de assegurar conhecimento e informações para dar condições para a vida digna dos cidadãos (Brasil, 1998).

Na perspectiva de orientar para a educação em saúde, apresentaram-se também nesta época, os temas transversais, que contam com um volume relativo a saúde. Nessa perspectiva, a saúde deixa de ser responsabilidade apenas da área de ciências da natureza e passa a ser considerado uma temática passível de ser abordada por todas as áreas, num viés transdisciplinar. Ou seja, além da presença do tema saúde nos conteúdos previstos para a área das Ciências da Natureza, seja para o Ensino Fundamental ou para o Ensino Médio, ele também é proposto como um tema transversal (Sousa, Guimarães \& Amantes, 2019).

No ano de 2013, foram apresentadas as Diretrizes Curriculares Nacionais da Educação Básica (DCNEB), com caráter normativo para a organização dessa etapa da escolarização, ressaltando a saúde como um campo do conhecimento a ser contemplado nas diferentes áreas. As mesmas reportam a saúde como elemento importante para a formação cidadã (Sousa; Guimarães \& Amantes, 2019).

Mais recentemente, observou-se a implementação do documento norteador, a BNCC, que "é um documento normativo que define o conjunto orgânico e progressivo de aprendizagens essenciais que todos os alunos devem desenvolver ao longo das etapas e modalidades da Educação Básica” (Brasil, 2017, p.7). Além disso, apresenta uma série de aportes teóricos que instituem e formalizam as orientações para o trabalho docente.

As competências que constam no documento expressam as aprendizagens mínimas que o aluno deve construir ao longo da escolaridade, focando na realidade e no letramento científico, visando a aplicação do conhecimento no cotidiano. Nesse sentido, a saúde é um tema cujos conceitos são importantes e necessários para a transformação da realidade, bem como compreensão dos recursos sociais e pessoais, bem como as capacidades físicas. Assim, não deve ser entendida como responsabilidade exclusiva do setor saúde, pois, a aprendizagem de um estilo de vida saudável, na direção de um bem-estar global passa pela educação. 
De acordo com Ilha, Righi, Rossi \& Soares (2013), as ações de promoção de saúde desenvolvidas na escola são um processo permanente, que têm como finalidade desenvolver conhecimentos, habilidades e destrezas para o autocuidado bem como a prevenção das condutas de risco. Nesse sentido, constata-se que a escola é um local por excelência que pode e deve abordar o tema saúde de modo que ocorra a disseminação de informações corretas com caráter preventivo e de manutenção da saúde.

Partindo do pressuposto de que a escola é um ambiente privilegiado para a abordagem de diversas temáticas importantes a população, e que a partir da organização de momentos em sala de aula para discutir e realizar projetos voltados a vida do estudante, apresentamos esse trabalho. Nessa perspectiva, o objetivo da pesquisa é verificar a presença do tema saúde na BNCC.

\section{Procedimentos Metodológicos}

Este estudo caracteriza-se como uma pesquisa documental, com objetivos exploratório, uma vez que busca descrever a presença (ou ausência) da temática saúde na BNCC (Gil, 2011).

Como principal suporte metodológico para a pesquisa documental, utilizou-se da análise de conteúdo temática para evidenciar unidades de registros relativos ao tema saúde constante no documento BNCC, relativo a Educação Infantil, Ensino Fundamental e Ensino Médio (Bardin, 2011). No estudo, as unidades de registros foram representadas por palavras e/ou palavras-chaves que estabeleciam relação direta com o tema saúde. Para tanto, a análise ocorreu em duas etapas.

A primeira etapa teve como objetivo verificar, ao longo da BNCC, a ocorrência da palavra "saúde" e as palavras-chaves: saudável; saudáveis; bem-estar; qualidade de vida; doença; prevenção; tratamento médico. A busca deu-se nos textos do documento, bem como, na parte específica dos componentes curriculares, conforme a Figura 1. 
Figura 1 - Estrutura da BNCC.

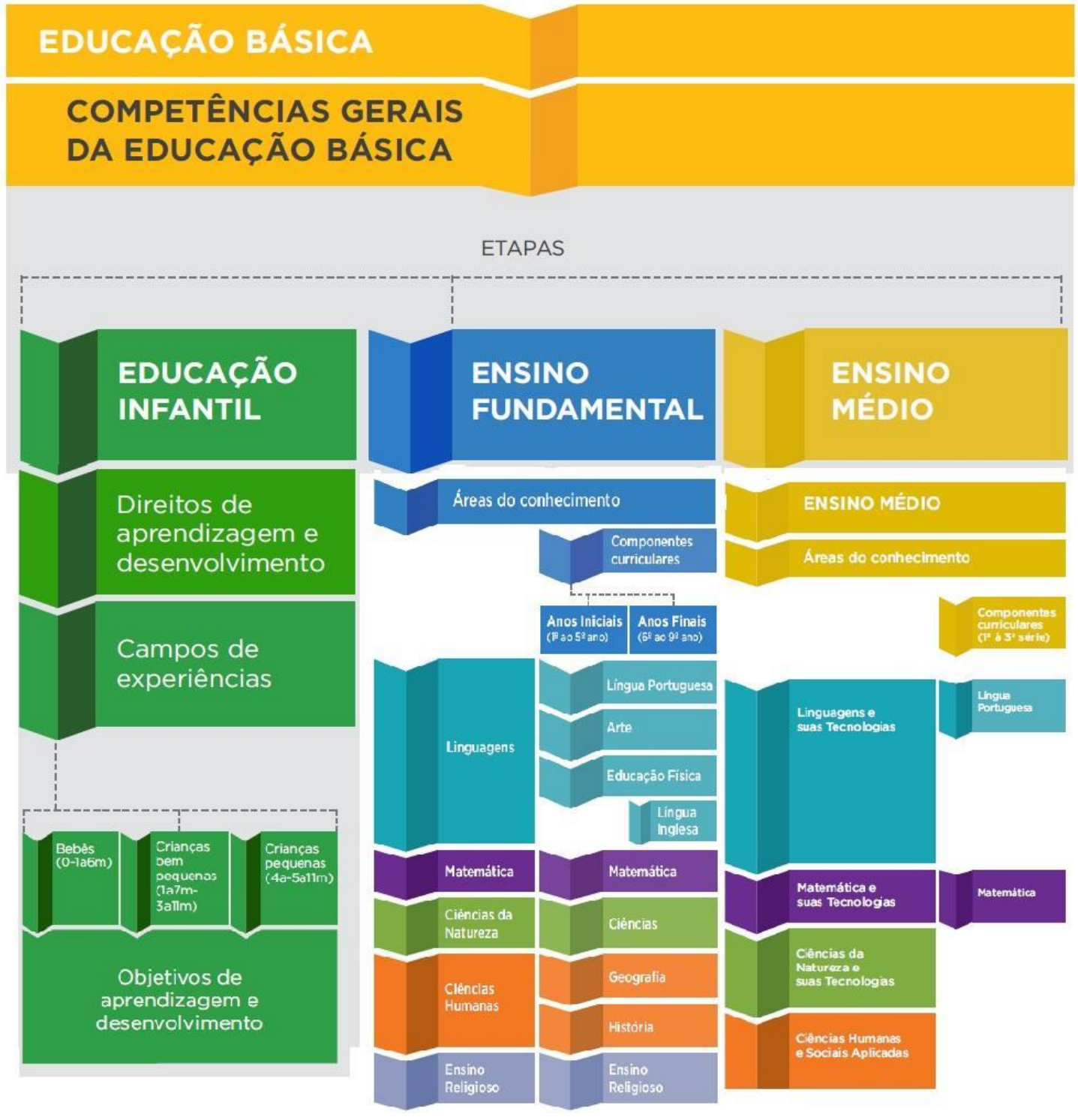

Fonte: Adaptado pelos autores (Brasil, 2017).

A busca da palavra-chave se deu a partir de duas formas: a leitura atenta e completa do documento e suas componentes curriculares, bem como pela busca automática através do localizador ctrl+f utilizando a palavra "saúde" neste procedimento. Foram levados em consideração os trechos/itens que abordassem a temática saúde e promoção da saúde de forma implícita e/ou explícita ao longo do parágrafo. As unidades de registro foram destacadas e os resultados foram descritos conforme frequência na área de conhecimento e nível de ensino onde constavam.

$\mathrm{Na}$ segunda etapa, as unidades de registro nos textos relacionados ao Ensino Fundamental passaram por leitura e análise de três pesquisadoras independentemente, a fim 
de constatar as concepções de saúde que emergiam dos extratos das unidades de registro. Posteriormente, os extratos do texto foram classificados em categorias. Ao final do processo, as análises foram trianguladas a fim de validar os resultados.

\section{Resultados e Discussão}

Para a análise da primeira etapa, que verificou ao longo da BNCC, a ocorrência da palavra "saúde" (S) e às palavras chaves: saudável (S); saudáveis (s); bem-estar (BE); qualidade de vida $(\mathrm{QV})$; doença ou doenças $(\mathrm{D})$; prevenção $(\mathrm{P})$; e tratamento médico $(\mathrm{T})$. $\mathrm{O}$ material foi extraído do documento oficial, foi sintetizado em um quadro onde o item foi sinalizado e remetido a componente curricular ao qual o item contempla (Figura 2).

Figura 2 - Incidência dos descritores ao longo da BNCC.

\begin{tabular}{|c|c|}
\hline Visão geral do documento & Ensino Fundamental \\
\hline $\begin{array}{l}\text { Saúde: } 60 \text { vezes }(\mathrm{S}) \\
\text { Saudáveis: } 4 \text { vezes (s) } \\
\text { Bem-estar: } 8 \text { vezes (BE) } \\
\text { Qualidade de Vida: } 18 \text { vezes (QV) } \\
\text { Doenças: } 8 \text { vezes (D) } \\
\text { Prevenção: } 5 \text { vezes (P) } \\
\text { Tratamento Médico: } 3 \text { vezes (T) }\end{array}$ & $\begin{array}{l}\text { Ed. Física: } 11 \text { vezes (S); } 1 \text { vez (BE); } 1 \text { vez (D) } \\
\text { Matemática: } 01 \text { vez (S); } 2 \text { vezes (T) } \\
\text { Ciências: } 24 \text { vezes (S); } 1 \text { vez (s); } 2 \text { vezes }(\mathrm{BE}) \text {; } \\
3 \text { vezes (QV); } 4 \text { vezes (D); } 4 \text { vezes (P); } 1 \text { vez } \\
\text { (T) } \\
\text { Geografia: } 01 \text { vez (S); } 12 \text { vezes (QV); } \\
\text { Ens. Religioso: } 03 \text { vezes (S); } 1 \text { vez (D) }\end{array}$ \\
\hline Educação Infantil & Ensino Médio \\
\hline $\begin{array}{l}\text { Saúde: } 1 \text { vez }(\mathrm{S}) \\
\text { Saudáveis: } 1 \text { vez }(\mathrm{s}) \\
\text { Bem-estar: } 2 \text { vezes (BE) }\end{array}$ & $\begin{array}{l}\text { Introdução: } 1 \text { vez }(\mathrm{S}) ; 1 \text { vez }(\mathrm{QV}) \\
\text { Linguagens e suas tecnologias: } 5 \text { vezes }(\mathrm{S}) \text {; } \\
1 \text { vez }(\mathrm{s}) ; 1 \text { vez }(\mathrm{BE}): 11 \text { vezes } \\
\text { Matemática: } 02 \text { vezes }(\mathrm{S}) \\
\text { Ciências da natureza: } 9 \text { vezes }(\mathrm{S}) ; 1 \text { vez } \\
(\mathrm{BE}): 2 \text { vezes }(\mathrm{OV}) ; 1 \text { vez }(\mathrm{P})\end{array}$ \\
\hline
\end{tabular}

Fonte: Os autores (2020).

Com essa análise constatou-se que a temática saúde na BNCC está voltada principalmente para o Ensino Fundamental, em detrimento da Educação Infantil, que será discutida mais adiante.

$\mathrm{Na}$ introdução do documento, a palavra saúde consta na oitava competência geral da 
educação básica: "Conhecer-se, apreciar-se e cuidar de sua saúde física e emocional, compreendendo-se na diversidade humana e reconhecendo suas emoções e as dos outros, com autocrítica e capacidade para lidar com elas" (Brasil, 2017, p.10), dando uma ideia de que é a promoção da saúde que fundamenta o texto. Enquanto competência geral, esperava-se que a temática saúde fosse visualizada nos textos relativos a todas as áreas do conhecimento, o que não foi verificado.

Na página 20, ainda no texto introdutório à $\mathrm{BNCC}$, relata-se que a temática da saúde e outras "são contempladas em habilidades dos componentes curriculares, cabendo aos sistemas de ensino e escolas, de acordo com suas especificidades, tratá-las de forma contextualizada" (Brasil, 2017, p.20). Novamente o documento incita que todas as componentes curriculares podem e devem trabalhar e desenvolver conhecimentos relativos à saúde.

A título de comparação, relata-se que a palavra saúde foi encontrada uma única vez ao longo do texto da Educação Infantil. Está alocada no item "Síntese das aprendizagens", no tópico: "Corpo, gestos e movimentos" onde discorre sobre o reconhecimento da "importância de ações e situações do cotidiano que contribuem para o cuidado de sua saúde e a manutenção de ambientes saudáveis" (Brasil, 2017, p. 54). Segundo Fernandes, Rocha e Souza (2005), a maior responsabilidade do processo de educação em saúde é a do professor, cabendo a este colaborar para o desenvolvimento do pensamento crítico do escolar, além de contribuir para que as crianças adotem comportamentos favoráveis à saúde.

A temática saúde foi encontrada com maior concentração nos textos relacionados aos anos finais do Ensino Fundamental, principalmente nas componentes curriculares de Ciências e Educação Física. Apesar de reconhecer que existe uma afinidade conceitual da temática saúde com essas componentes curriculares, entendemos que as demais componentes também podem contribuir para o desenvolvimento de conhecimentos e competências em saúde, numa visão transdisciplinar para desenvolver a cidadania e autonomia dos indivíduos. A visão transdisciplinar pode auxiliar na desfragmentação dos currículos e para os professores realizar um trabalho coletivo entre as componentes. O tema saúde possibilita envolver alunos, contextualizando e promovendo a construção de conhecimentos coletivamente.

Daí a importância da transdisciplinaridade nutrida por uma visão complexa da realidade como atitude epistemológica, como princípio e como metodologia aberta de construção do conhecimento, como ferramenta capaz de assegurar o espaço de interconexão disciplinar, de uma educação intercrítica e intercultural, nutrida por uma pluralidade de olhares, linguagens, compreensões e percepções da realidade que destroem todo e qualquer dogmatismo, fundamentalismo e pensamento unívoco (Moraes, 2018, p.30). 
As componentes curriculares de Matemática, História, Geografia, Língua Portuguesa, Língua Inglesa, Artes e Ensino Religioso não reconhecem a temática saúde como possível objeto de conhecimento. Se considerarmos a possibilidade e importância de contextualização dos conceitos e o estudo a partir de temas socialmente relevantes, entende-se que esses componentes curriculares perdem a oportunidade de desenvolver seus conceitos por meio da temática saúde. Ou seja, a interdisciplinaridade não transpassa essas componentes e pode ser apontada como uma fragilidade da BNCC.

Uma vez que a potencialidade do desenvolvimento de um currículo interdisciplinar possibilita "[...] ações disciplinares sobre um determinado tema, sendo que são articuladas por meio de um conjunto de atividades coordenadas que tem como meta a construção de um objetivo em comum" (Lima; Kraisig; Saccomori; Catardo \& Reis, 2017 p.37). Isso resultará em um rompimento das fronteiras disciplinares e possibilita ao aluno uma visão de mundo integrada articulando o conhecimento sobre a saúde podendo aplicá-la em sua vida e da comunidade.

Como a BNCC não apresenta essa característica se torna ainda mais fragmentado os currículos, não sendo obrigatório a vinculação das disciplinas. O que para os alunos é uma regressão, onde a visão de mundo e sua aplicabilidade na vida cotidiana se torna de difícil entendimento.

A fim de evitar a possibilidade de viés de análise, realizou-se buscas utilizando outras palavras relacionadas a temática saúde (Figura 2), como "saudável” (não encontrado no documento); saudáveis (s) (4 vezes); bem-estar(BE) (8 vezes); qualidade de vida(QV) (18 vezes); “doença” (D) (8 vezes); "prevenção”(P) (5 vezes) e tratamento médico (T) (3 vezes).

Com relação a palavra saudáveis (s), está inserida na Educação Infantil como síntese de aprendizagem, no Ensino Fundamental está restrito a componente curricular de Ciências, e no que se refere ao Ensino Médio, consta na introdução e na área do conhecimento Linguagens e suas tecnologias. Sobre a palavra bem-estar (BE), ela esteve presente em todos os seguimentos da Educação Básica, na Educação Infantil aparece como objetivos de aprendizagem e síntese das aprendizagens e se apresentou como uma habilidade no Ensino Fundamental no componente curricular de Educação Física e como competência na componente curricular de Ciências. Já no Ensino Médio apresentou-se como habilidade na área de Ciências da Natureza e suas tecnologias.

Quanto a palavra qualidade de vida (QV) está ausente na Educação Infantil, e se apresenta como habilidade no componente curricular de Ciências do Ensino Fundamental e como unidade temática em Geografia. No que se refere ao Ensino Médio, a palavra qualidade 
de vida está inserida no texto introdutório que fala a respeito dessa etapa de vida escolar e como uma habilidade na área de Ciências da Natureza e suas tecnologias.

Foi possível verificar que a utilização da palavra doença(s) está estritamente relacionada à ausência de saúde, com enfoque em possibilitar ao aluno conhecer quais as doenças comumente acometem o ser humano. A palavra doença foi encontrada no Ensino Fundamental como habilidades nas componentes curriculares de Educação Física, Ciências e Ensino Religioso. Na Educação Física, a palavra está ao lado da palavra saúde como competência específica. Salienta-se ainda, que está presente no texto introdutório da Ciências Sendo ausente no Ensino Médio e na Educação Infantil.

Ainda que a saúde como ausência de doença seja a concepção mais divulgada, penso ser especialmente importante não nos esquecermos que, segundo a OMS (Organização Mundial de Saúde), ser saudável significa viver em equilíbrio e em bem-estar biopsicosocial e espiritual (Oliveira, 2005, p.2).

Outro ponto a ser considerado, é sobre o eixo prevenção (P). Este foi percebido 4 vezes no Ensino Fundamental e apenas 1 vez no Ensino Médio. Quanto a sua localização, sua inserção está em ambas etapas de ensino, diretamente relacionado a componente curricular de Ciências, no qual ressalta a importância do trabalho do professor na disseminação de informações corretas e ações que evitem às doenças, resultando em uma sensibilização por parte dos alunos nas escolhas corretas.

A palavra-chave tratamento médico (T) é sinalizada 3 vezes no documento e suas aparições são ao longo do Ensino Fundamental. Destas, 1 vez no texto introdutório da componente de Ciências e as outras duas vezes na componente de matemática como habilidade, porém referindo-se a mesma habilidade. A habilidade está relacionada a identificar situações do cotidiano nas quais seja necessário a tomada de decisões considerando os riscos probabilísticos (optar por este ou outro tratamento médico, por exemplo).

Sabe-se que a abrangência do termo saúde vai muito além de informação, instrução e divulgação de dados que possam prevenir o surgimento de algumas doenças. O termo saúde também pode estar vinculado a construção de projetos, atividades que envolvam às necessidades da comunidade que cerca a escola, bem como a investigação do ambiente em que vivem, das pessoas que o cercam e quais as possibilidades de intervenção na localidade onde mora.

O conceito pela OMS de saúde, diferente do conceito de saúde como ausência de doença, se preocupa com as diferentes ordens de complexidade dos fatores envolvidos como o processo saúde/doença, como por exemplo a ausência de saúde como o resultado de uma teia 
de condicionantes sociais, econômicos, políticos, culturais, ambientais, comportamentais, psicológicos e biológicos (Barros, Martorelli \& Freitas, 2005).

Visto que, a saúde está relacionada ao bem-estar do corpo anatomicamente, o componente curricular de Ciências se destaca pelo trabalho desenvolvido com a temática saúde devido a sua estruturação curricular na formação inicial, onde o professor de Ciências perpassa pelas disciplinas relacionadas a saúde/corpo. Muitos professores admitem que o ensino de tais temas, como o da saúde, é tarefa exclusiva dos professores de Ciências devido à proximidade com o tema (Costa, Gomes \& Zancul, 2011).

No entanto, os temas relacionados à saúde não devem estar circunscritos em um único componente, mas sim deve apresentar um caráter globalizador. Assim, é importante que todos os professores, dos diversos componentes, se envolvam e participem de atividades que tenham como objetivo o desenvolvimento de atitudes e reflexão a respeito das questões relativas à saúde dos alunos.

\section{Considerações Finais}

O objetivo deste trabalho foi verificar a presença do tema saúde na BNCC. Podemos afirmar que a temática saúde ainda está confinada nas componentes curriculares de Ciências e Educação Física, sendo rara a utilização do termo ou do conceito em outros componentes curriculares. Isso mostra-se como uma fragilidade ao trabalho interdisciplinar e contextualizado da prática pedagógica.

Por outro lado, percebe-se que a perspectiva de saúde abordada na componente de ciências é extremamente biológica e com enfoque na diminuição dos riscos à doença. Ou seja, aspectos relacionados à saúde ambiental, social ou mesmo cultural não fazem parte dos objetos de conhecimento desta área. No que se refere a Educação Física, o panorama não muda muito. A perspectiva de saúde adotada faz referência a estética e cuidado do corpo, e por sua vez, também não tem a preocupação com questões ambientais e sociais que podem interferir na saúde da população.

Dessa forma, entendemos a necessidade de que as escolas discutam e incrementem seus currículos com o tema saúde, visto que está presente como tema transversal nos Parâmetros Curriculares Nacionais e aparentemente perde sua notoriedade ao longo da BNCC. Cabe ainda, a realização de outro(s) estudo(s) que apresentem as perspectivas de condicionantes relacionadas à temática, nos sentidos: ambientais, psicossociais, culturais, socioeconômicas e biológicas. 
Além disso, também é importante que as universidades e mantenedoras promovam ações que deem atenção especial na produção de material e/ou sequências didáticas que possam ser implementadas na escola básica. Que sirvam de apoio aos professores para a construção de estratégias didáticas que auxiliem na ampla conscientização sobre a temática.

Esse trabalho não encerra a discussão sobre a BNCC e outras leituras e análises podem ser realizadas. Entende-se que sua implementação e os efeitos dela possam ser acompanhados e pesquisados, de forma a descrever os efeitos da mesma na formação dos indivíduos para a cidadania.

\section{Referências}

Barbi, JSP \& Neto, JM. (2017). A Saúde nos anos finais do Ensino Fundamental: Uma análise de documentos de referência. XI Encontro Nacional de Pesquisa em Educação em CiênciasXI ENPEC. Universidade Federal de Santa Catarina, Florianópolis, SC-3 a, 6.

Barros, CMS, Martorelli, RDCG. \& Freitas, VVD. (2006). Modelo da atividade educação em saúde. In Modelo da atividade educação em saúde.

Brasil. (1971). Lei n 5.692 , de 11 de agosto de 1971. Fixa diretrizes e bases para o ensino de $1^{\circ}$ e $2^{\circ}$ graus, e dá outras providências. Diário Oficial da União.

Brasil. (1998). Parâmetros Curriculares Nacionais: terceiro e quarto ciclos: apresentação dos temas transversais. Secretaria de Educação Fundamental. Brasília: MEC/sef, 1998, 156.

Brasil. (2017). Base Nacional comum curricular. Ministério da Educação. Brasília-DF: MEC, Secretaria de Educação Básica, 2017.

Costa, S, Gomes, PHM. \& ZANCUL, MD. (2011). Educação em Saúde na escola na concepção de professores de Ciências e de Biologia. Núcleo de Educação Científica. Brasília.

Fernandes, MH, Rocha, VM. \& Souza, DBD. (2005). A concepção sobre saúde do escolar entre professores do ensino fundamental ( $1^{\mathrm{a}}$ a $4^{\mathrm{a}}$ séries). História, Ciências, Saúde-

Manguinhos, 12(2), 283-291. 
Gil, AC. (2010). Como elaborar projetos de pesquisa. São Paulo: Atlas, 2006.

Ilha, PV, Righi, MMT, Rossi, DS. \& Soares, FAA. (2013). A promoção da saúde nos livros didáticos de ciências do $6^{\circ}$ ao $9^{\circ}$ ano. Alexandria: Revista de Educação em Ciência e Tecnologia, 6(3), 107-120.

Lima, APS, Kraisig, ÂR, Saccomori, F, Catardo, LS \& Reis, MT. (2017) A interdisciplinaridade como ferramenta de integração. In: Pessano, EFC, Querol, MVM, Lima, APS \& Castro, LRB. Contribuições para o ensino de ciências: Alfabetização Científica, Aprendizagem Significativa, Contextualização e Interdisciplinaridade. Bagé/RS:

EdUNIPAMPA, p. 34-46.

Oliveira, CC. (2005). Como educar para a saúde? Disponível em:

https://repositorium.sdum.uminho.pt/bitstream/1822/13648/1/P\%C3\%BAblico.pdf. Acessado em: 2 abr. 2020.

Monteiro, PHN. \& Bizzo, N. (2015). A saúde na escola: análise dos documentos de referência nos quarenta anos de obrigatoriedade dos programas de saúde, 1971-2011. História, Ciências, Saúde-Manguinhos, 22(2), 411-428.

Moraes, MC. (2018). Transdisciplinaridade, criatividade e educação: fundamentos ontológicos e epistemológicos. Papirus Editora.

Sousa, MC, Guimarães, APM. \& Amantes, A. (2019). A saúde nos documentos curriculares oficiais para o ensino de ciências: da lei de diretrizes e bases da educação à base nacional comum curricular. Revista Brasileira de Pesquisa em Educação em Ciências, 129-153.

World Health Organization. (2011). Constituição da Organização Mundial da Saúde (OMS/WHO). 1946. 
Research, Society and Development, v. 9, n. 7, e509974457, 2020

(CC BY 4.0) | ISSN 2525-3409 | DOI: http://dx.doi.org/10.33448/rsd-v9i7.4457

Porcentagem de contribuição de cada autor no manuscrito

Camila Pereira Burchard - 20\%

Renata Godinho Soares $-20 \%$

Verônica de Carvalho Vargas - 20\%

Phillip Vilanova Ilha $-20 \%$

Raquel Ruppenthal - 20\% 